Carnegie Observatories Astrophysics Series, Vol. 2:

Measuring and Modeling the Universe, 2004

ed. W. L. Freedman (Cambridge: Cambridge Univ. Press)

\title{
A Brief History of Cosmology
}

\author{
MALCOLM S. LONGAIR \\ Cavendish Laboratory, Cambridge, UK
}

\begin{abstract}
Some highlights of the history of modern cosmology and the lessons to be learned from the successes and blind alleys of the past are described. This heritage forms the background to the lectures and discussions at this Second Carnegie Centennial Symposium, which celebrates the remarkable contributions of the Carnegie Institution in the support of astronomical and cosmological research.
\end{abstract}

\subsection{Introduction}

It is a great honor to be invited to give this introductory address at the Second Carnegie Centennial Symposium to celebrate the outstanding achievements of the Observatories of the Carnegie Institution of Washington. I assume that the point of opening this meeting with a survey of the history of cosmology is not only to celebrate the remarkable achievements of modern observational and theoretical cosmology, but also to provide lessons for our time, which may enable us all to avoid some of the errors that we now recognize were made in the past. I am bound to say that I am not at all optimistic that this second aim will be achieved. I recall that, when I gave a similar talk many years ago with the same intention, Giancarlo Setti made the percipient remark:

Cosmology is like love; everyone likes to make their own mistakes.

By its very nature, the subject involves the confrontation of theoretical speculation with cosmological observations, the scepticism of the hardened observer about taking anything a theorist says seriously, the problems of pushing observations to the very limits of technological capability, and sometimes beyond these, resulting in dubious data, and so on. These confrontations have happened many times in the past. My suspicion is that the community of astronomers and cosmologists is now sufficiently large for false dogma and insecure observations to have only limited shelf-lives, but we must remain vigilant. Nonetheless, it is intriguing to survey the present state of cosmology, with its extraordinary successes and challenges, and recognize the many similarities to those that faced the great scientists of the past. I leave it to readers to draw their own preferred analogies.

The history of cosmology is a vast and fascinating subject, and I will only touch on some of the highlights of that story. I have given a more detailed account of that history elsewhere (Longair 1995), and it is a subject that repays careful study. To my regret, there will be little space to do justice to the technological achievements that have made modern cosmology a 


\section{S. Longair}

rather exact science (see Longair 2001). Without these developments, none of us would be celebrating the achievements of modern cosmology at this symposium.

Before getting down to the history, let me contribute a personal appreciation of Andrew Carnegie's philanthropy. He gave away $\$ 350 \mathrm{M}$ of his fortune of $\$ 400 \mathrm{M}$ to charitable causes. Among the more remarkable of these was the founding of about 3000 libraries worldwide, including five in my home town of Dundee in Scotland, only about 30 miles from Dunfermline, Carnegie's birthplace. Here is a quotation from the Carnegie Libraries of Scotland web-site:

When the library was officially opened on 22 October 1908, [Charles] Barrie [a former Lord Provost] was asked to perform the opening ceremony... There was a banquet afterwards in the Victoria Art Galleries hosted by Lord Provost Longair.

Lord Provost Longair was my great, great uncle. I remember as a small boy going regularly to the Dundee Public Libraries to learn about rockets and space flight. Little did I realise then that, more than 50 years later, I would be participating in the celebrations of the centenary of the founding of the Carnegie Institution.

\subsection{Observational Cosmology to 1929}

The earliest cosmologies of the modern era were speculative cosmologies. The "island universe" model of Descartes, published in The World of 1636, involved an interlocking jigsaw puzzle of solar systems. Wright's An Original Theory of the Universe of 1750 involved spheres of stars and solar systems, while Kant in 1755 and Lambert in 1761 developed the first hierarchical, or fractal, pictures of the Universe (see Harrison 2001). The problem with these early cosmologies was that they lacked observational validation. When these ideas were put forward, the only star whose distance was known was the Sun. The first parallax measurements of stars were only made in the 1830s by Friedrich Bessel, Friedrich Georg Wilhelm Struve and Thomas Henderson.

The first quantitative estimates of the scale and structure of the Universe were made by William Herschel in the late 18th century. Herschel's model of the large-scale structure of the Universe was based upon star counts and provided the first quantitative evidence for the "island universe" picture of Wright, Kant, Swedenborg and Laplace. In deriving his famous model for our Galaxy, Herschel assumed that all stars have the same absolute luminosities. The importance of interstellar extinction in restricting the number counts of stars to a relatively local region of our Galaxy was only fully appreciated in the early 20th century.

John Michell had already warned Herschel that the assumption that the stars have a fixed luminosity was incorrect. This is the same John Michell who was Woodwardian Professor of Geology at Queen's College, Cambridge, before becoming the rector of Thornhill in Yorkshire in 1767. He designed and built what we now know as the Cavendish experiment to measure the mean density of the Earth. Nowadays, he is rightly remembered as the inventor of black holes. In 1767, he showed that there must be a dispersion in the absolute luminosities of the stars from observations of bright star clusters. Despite this warning, Herschel ignored the problem and proceeded to produce a number of different versions of the structure of our Galaxy. In 1802, Herschel measured the magnitudes of visual binary stars and was forced to agreed with Michell's conclusion. Equally troubling was the fact that observations with his magnificent 40 -foot telescope showed there was no edge to the 


\section{S. Longair}

Galaxy. He continued to find stars the fainter he looked-evidently, the stellar system was unbounded. Eventually, Herschel lost faith in his model of the Galaxy.

The desire to observe the Universe with telescopes of greater and greater aperture continued throughout the nineteenth century. The largest reflecting telescope constructed during that century was the great 72-inch reflector at Birr Castle in Ireland by William Parsons, the 3rd Earl of Rosse. This "Great Leviathan" was moved by ropes and astronomical objects could be tracked by moving the barrel of the telescope between the two large walls, which also accommodated a movable observing platform at the Newtonian focus of the telescope. Observations were made by eye and so the "length of the exposure" was limited to about a tenth of a second. Despite the difficulties of making observations and the inclement weather in central Ireland, Lord Rosse was able to resolve nebulae into stars and, perhaps most important of all, discovered the spiral structure of galaxies, the most famous drawing being his sketch of M51.

The revolutions that led to the discipline of extragalactic astronomy as we know it today were the use of photography to record astronomical images and the shift from refracting to reflecting telescope designs. The Yerkes 40 -inch refractor was the end of the line so far as refracting telescopes were concerned. The much more compact reflecting design had the advantage of greater collecting area, but was much more sensitive to tracking and guiding errors. Many key technologies were developed during the latter half of the nineteenth century, thanks to pioneers such as Lewis Morris Rutherfurd, John Draper, Andrew Common and George Carver. These pioneers solved the problems of the tracking and pointing of reflecting telescopes, an invention of particular importance being the adjustable plate holder, which enables the observer to maintain the pointing of the telescope with high precision.

The resulting technical advances contributed to the remarkable achievement of James Keeler and his colleagues at the Lick Observatory in enhancing the performance of the 91$\mathrm{cm}$ Crossley reflector to become the premier instrument for astronomical imaging. During the commissioning of the Crossley reflector in 1900, Keeler obtained spectacular images of spiral nebulae, including his famous image of M51. Not only were the details of its spiral structure observed in unprecedented detail, but there were also large numbers of fainter spiral nebulae of smaller angular size. If these were objects similar to the Andromeda Nebula M31, they must lie at very great distances from our Solar System. Tragically, just as this new era of astronomy was dawning, Keeler died of a stroke later that same year at the early age of only 42 (Osterbrock 1984).

George Ellery Hale plays a central role in the celebrations of the centenary of the Carnegie Institution. He is rightly regarded as the most successful astronomical entrepreneur of the modern era. He maintained an unswerving determination to construct successively larger and larger telescopes from the time of his directorship of the Yerkes Observation in the 1890s through the period when he became Director of the Mount Wilson Observatory in 1903 until his death in 1938. In 1895, he had persuaded his father to buy the 1.5 meter blank for a 60-inch reflecting telescope. The design was to be an enlarged version of the Calver-Common design for the 91-cm Crossley reflector at the Lick Observatory. Before the 60 -inch telescope was completed, however, he persuaded J. D. Hooker to fund an even bigger telescope, the 100-inch telescope to be built on Mount Wilson. The technological challenges were proportionally greater, the mass of the telescope being 100 tons, but the basic CalverCommon design was retained. The optics were the responsibility of George Ritchey, an optical designer of genius, who was to come up with the ingenious optical configuration 


\section{S. Longair}

known as the Ritchey-Chrétien design, which enabled excellent imaging to be achieved over a wide field of view. The 60- and 100-inch telescopes became the prime telescopes for the study of the spiral nebulae, but these accomplishments were not achieved without an enormous effort on Hale's part.

The story of Hale's construction of these telescopes is a heroic tale. Equally impressive is Andrew Carnegie's generosity in enabling Hale to realise his vision. Carnegie's fateful visit to the Mount Wilson Observatory in 1906 has been recorded in the volume The Legacy of George Ellery Hale (Wright, Warnow, \& Weiner 1972). Carnegie was clearly impressed by what he saw during his visit. As recorded in the local Pasadena newspaper, he remarked:

"We do not know what may be discovered here," he said. Franklin had little idea what would be the result of flying his kite. But we do know that this will mean the increase of our knowledge in regard to this great system of which we are part.

Mr. Hale has discovered here 1600 worlds about one of the stars which were not known before. We have found helium in the Sun, and after finding it there, we find it in the Earth. It all goes to show that all things are of a common origin.

To anyone who has had the fortune to be responsible for the operation of a large observatory facility, these remarks have heartening resonances. Carnegie did not quite get the science right so far as the stars were concerned, but he got it absolutely right so far as helium is concerned. Helium was discovered astronomically long before it was identified in the laboratory and is but one of many examples of how astronomical observations can provide key insights into the behavior of matter under circumstances which are only later reproduced in the laboratory. Plainly, Hale had carried out a very successful campaign in enthusing Carnegie about the importance of progress in astronomy.

Following his visit, Carnegie pledged an additional $\$ 10 \mathrm{M}$ to the endowment of the Carnegie Institution, specifically requesting that the benefaction be used to enable the work of the Observatory to proceed as rapidly as possible. This is the purist music to the ears of any Observatory Director, who knows that, while it is usually possible in the end to find the capital resources for ambitious projects, these cannot succeed without matching funds for operations in the long term. Carnegie's vision and understanding are models for benefactors of astronomy.

The construction of the 60- and 100-inch telescopes were stressful and Hale suffered a nervous breakdown in 1910. It is touching to read Carnegie's letter to Hale of 1911 (with the original spelling), urging him to take care of his health:

November 27, 1911

Mr dear Frend,-

Delited to read your long note this morning; not too long-every word tells, but pray show your good sense by keeping in check your passion for work, so that you may be spared to put the capstone upon your career, which should be one of the most remarkable ever lived.

Ever yours,

Andrew Carnegie.

Carnegie's benefaction was crucial for the completion of the 100-inch Hooker Telescope at Mount Wilson. The telescope was by far the largest in the world and incorporated all the lessons learned from the works of earlier telescope builders. Completed in 1918, this 


\section{S. Longair}

instrument was to dominate observational cosmology for the next 30 years until the commissioning of the Palomar 200-inch telescope in 1948.

The Hooker 100-inch telescope played a central role in the resolution of what became known as the "Great Debate," which concerned the related issues of the size of our own Galaxy and the nature of the white, or spiral, nebulae. This confrontation between Harlow Shapley and Heber D. Curtis is too well known to need much amplification here (see Christianson, this volume). In 1899, Scheiner had obtained a spectrogram of M31 and stated that the spectrum suggested a "cluster of Sun-like stars." In 1922, Öpik estimated the distance of M31 by comparing the mass-to-light ratio of the central region of M31 with that of our own Galaxy and found a distance of $440 \mathrm{kpc}$, suggesting that it lay well outside the confines of our Galaxy. The discovery of variable stars in spiral nebulae by Duncan in 1922 led to a flurry of activity and Hubble's famous discovery of Cepheid variables in M31.

Central to Hubble's use of Cepheid variable stars in M31 to measure its distance was the discovery of the period-luminosity relation for Cepheids in the Magellanic Clouds by Henrietta Leavitt (Leavitt 1912). Leavitt, like Annie Cannon, was profoundly deaf. While she is best remembered for her work on the Cepheid variables, her main work was the establishment of the North Polar Sequence, the accurate determination of the magnitude scale for stars in a region of sky which would always be accessible to observers in the Northern Hemisphere. By the time of her death in 1921, she had extended the North Polar Sequence from 2.7 to 21 st magnitude, with errors less than 0.1 magnitudes. To achieve this, she used observations from 13 telescopes ranging from 0.5 to 60 inches in diameter and compared her scale using 5 different photographic photometric techniques. Without this fundamental work, the magnitude scale for galaxies could not have been established.

It is intriguing that by far the most stubborn pieces of observational evidence against what might be termed the long distance scale were van Maanen's measurements of the proper motions of spiral arms. It is now well understood how difficult it is to measure tiny displacements of any diffuse object—van Maanen's evidence was only refuted in 1933 by Edwin Hubble after a considerable observational effort.

Hubble's paper of 1925 establishing the extragalactic nature of the spiral nebulae is impressive enough (Hubble 1925), but to my mind his paper of the following year entitled Extragalactic Nebulae is even more compelling (Hubble 1926). In this paper, he provided the first more or less complete description of galaxies as extragalactic systems. The paper includes a morphological classification of galaxies into the classic Hubble types, estimates of the relative numbers of different types, estimates of mass-to-luminosity ratios for different types of galaxies and their average number densities. Finally, the mean mass density in galaxies in the Universe as a whole was derived for the first time. Adopting Einstein's static model for the Universe, the radius of curvature of the spherical geometry was $\mathcal{R}=27,000$ $\mathrm{Mpc}$ and the total number of galaxies $3.5 \times 10^{15}$. Thus, by 1926, the first application of the ideas of relativistic cosmology to the Universe of galaxies had been made. Hubble concluded that the observations already extended to about $1 / 300$ of the radius of the closed Einstein universe. The prophetic last sentence of his great paper reads

... with reasonable increases in the speed of the plates and size of telescopes, it may become possible to observe an appreciable fraction of the Einstein Universe.

It is no surprise that Hale began his campaign to raise funds for the 200 -inch telescope in 


\section{S. Longair}

1928. By the end of the year, he had received the promise of a grant of $\$ 6 \mathrm{M}$ from the Rockefeller foundation.

Hubble's important insights were soon followed by an even more remarkable discovery. In 1917, Vesto M. Slipher had published his heroic pioneering spectroscopic observations of 25 spiral nebulae (Slipher 1917). He realised that, for the spectroscopy of low surface brightness objects such as the spiral nebulae, the crucial factor was the $f$-ratio of the spectrograph camera, not the size of the telescope. The observations involved very long integrations of 20,40 or even 80 hours with small telescopes.

The velocities of the galaxies inferred from the Doppler shifts of their absorption lines were typically about $570 \mathrm{~km} \mathrm{~s}^{-1}$, far in excess of the velocity of any known object in our Galaxy. Furthermore, most of the velocities corresponded to the galaxies moving away from the Solar System, that is, the lines were redshifted to longer (red) wavelengths. In 1921, Carl Wilhelm Wirtz concluded that, when the data were averaged in a suitable way, "an approximate linear dependence of velocity upon apparent magnitude is visible" (Wirtz 1921). By 1929, Hubble had assembled approximate distances of 24 galaxies for which velocities had been measured, mostly by Slipher, all within $2 \mathrm{Mpc}$ of our Galaxy. I have always been impressed that Hubble was able to find the law which bears his name from the very crude distance indicators which he had available. The first seven objects within 500 kpc had Cepheid distances; the distances of the next 13 were found assuming the brightest stars all had the same absolute magnitude; the last four, in the Virgo cluster, were estimated on the basis of the mean luminosities of nebulae in the cluster. From these meager data, Hubble derived his famous redshift-distance relation (Hubble 1929). If the redshifts $z$ are interpreted as the Doppler shifts of galaxies due to their recession velocities $v$, the relation can be written $v=H_{0} r$, where $H_{0}$ is Hubble's constant.

Milton Humason had by then mastered the use of the 100-inch telescope for obtaining the spectra of faint galaxies and by 1934 Humason and Hubble had extended the velocitydistance relation to $7 \%$ of the speed of light (Humason \& Hubble 1934). Furthermore, Hubble realised that he could test for the isotropy and homogeneity of the Universe by counting the numbers of faint galaxies. Hubble established that the numbers of galaxies increased with increasing apparent magnitude in almost exactly the fashion expected if they were uniformly distributed in space.

Even before 1929, however, it was appreciated that Hubble's law was expected according to world models based upon the general theory of relativity.

\subsection{Theoretical Cosmology to 1939}

Let us turn to theoretical cosmology and the history of Einstein's static model for the Universe. Working independently, Lobachevsky in Kazan in Russia and Bolyai in Hungary solved the problem of the existence of geometries that violated Euclid's fifth axiom in 1825. These were the first self-consistent hyperbolic (non-Euclidean) geometries. In his great text On the Principles of Geometry (1825), Lobachevsky worked out the minimum parallax of any star in hyperbolic geometry

$$
\theta=\arctan \left(\frac{a}{\mathcal{R}}\right)
$$

where $a$ is the radius of the Earth's orbit and $\mathcal{R}$ the radius of curvature of the geometry. In his textbook, he found a minimum value of $\mathcal{R} \geq 1.66 \times 10^{5} \mathrm{AU}$. What is intriguing is that this 


\section{S. Longair}

estimate was made 8 years before Bessell's announcement of the first successful parallax measurement of 61 Cygni. In making his estimate, Lobachevsky used the observational upper limit to the parallax of bright stars. In a statement which will warm the heart of observational astronomers, and which is particularly apposite in the light of what we will hear at this meeting, he remarked

There is no means other than astronomical observations for judging the exactness which attaches to the calculations of ordinary geometry.

The pioneering works of Lobachevsky and Bolyai led to Riemann's introduction of quadratic differential forms, his generalization of their results to non-Euclidean geometries, and his discovery of spaces of positive curvature - that is, spherical non-Euclidean geometries.

Unlike his other great discoveries, Einstein's route to general relativity was long and tortuous. Four ideas were important in his search for a self-consistent relativistic theory of gravity:

- The influence of gravity on light

- The principle of equivalence

- Riemannian spacetime

- The principle of equivalence

Toward the end of 1912, he realised that what was needed was non-Euclidean geometry. Einstein consulted his old school friend, Marcel Grossmann, about the most general forms of transformation between frames of reference for metrics of the form

$$
\mathrm{d} s^{2}=g_{\mu \nu} \mathrm{d} x^{\mu} \mathrm{d} x^{\nu} .
$$

Grossmann soon came back with the answer that the most general transformation formulae were the Riemannian geometries, but that they had the "bad feature" that they are nonlinear. Einstein instantly recognized that, on the contrary, this was a great advantage since any satisfactory theory of relativistic gravity must be nonlinear.

After further years of struggle, during which he and Grossmann were ploughing very much a lone furrow, general relativity was formulated in its definitive form in 1915 (Einstein 1915). In 1916, Willem de Sitter and Paul Ehrenfest suggested in correspondence that a spherical 4-dimensional spacetime would eliminate the problems of the boundary conditions at infinity, which pose insuperable problems for Newtonian cosmological models. In 1917, Einstein realised that, in general relativity, he had for the first time a theory which could be used to construct fully self-consistent models for the Universe as a whole (Einstein 1917). At that time, the expansion of the Universe had not been discovered.

One of objectives of Einstein's program was to incorporate into the structure of general relativity what he called Mach's Principle, meaning that the local inertial frame of reference should be determined by the large-scale distribution of matter in the Universe. There was, however, a further problem, first noted by Newton, that static model universes are unstable under gravity. Einstein proposed to solve both problems by introducing an additional term into the field equations, the cosmological constant $\Lambda$. In Newtonian terms, the cosmological constant corresponds to a repulsive force $\vec{f}$ acting on a test particle at distance $\vec{r}, \vec{f}=\frac{1}{3} \Lambda \vec{r}$. Unlike gravity, this force is independent of the density of matter. The $\Lambda$-term has negligible influence on the scale of the Solar System and is only appreciable on cosmological scales.

The equation that describes the expansion becomes 


\section{S. Longair}

$$
\frac{\mathrm{d}^{2} R}{\mathrm{~d} t^{2}}=-\frac{4 \pi G \rho_{0}}{3 R^{2}}+\frac{1}{3} \Lambda R
$$

The first term on the right-hand side describes the deceleration due to gravity and the second what Zel'dovich referred to as the "repulsive effect of the vacuum" (Zel'dovich 1968). At that time, the physical significance of the $\Lambda$ term was not understood.

Einstein believed that he had incorporated Mach's Principle into general relativity. In his words,

The inertial structure of spacetime was to be "exhaustively conditioned and determined" by the distribution of material throughout the Universe.

Further, he stated that the extension of the field equations was "not justified by our actual knowledge of gravitation," but was "logically consistent." Furthermore, the cosmological term was "necessary only for the purpose of making possible a quasi-static distribution of matter, as required by the fact of the small velocities of stars."

From Einstein's field equations of general relativity, it followed that the geometry of Einstein's static universe is closed and the radius of curvature of the geometrical sections is $\mathcal{R}=c /\left(4 \pi G \rho_{0}\right)^{1 / 2}$, where $\rho_{0}$ is the mean density of the static Universe. The value of $\Lambda$ was directly related to the mean density of the Universe, $\Lambda=4 \pi G \rho_{0}$. Einstein believed that he had incorporated Mach's Principle into general relativity, in that static solutions of the field equations did not exist in the absence of matter.

Almost immediately, de Sitter (1917) showed that one of Einstein's objectives had not been achieved. He found solutions of Einstein's field equations in the absence of matter, $\rho=p=0$. The metric he derived had the form

$$
\mathrm{d} s^{2}=\mathrm{d} r^{2}-R^{2} \sin \left(\frac{r}{R}\right)\left(\mathrm{d} \phi^{2}+\cos ^{2} \phi \mathrm{d} \theta^{2}\right)+\cos ^{2}\left(\frac{r}{R}\right) c^{2} \mathrm{~d} t^{2}
$$

Although there is no matter present in the Universe, a test particle still moves along a perfectly well-defined path through spacetime. As de Sitter remarked, "If no matter exists apart from the test body, has this inertia?" One prediction of de Sitter's paper was the fact that distant galaxies would be observed with a redshift, although in his solution the metric was stationary-this phenomenon became known as the de Sitter effect.

In 1922, Kornel Lanczos showed that by a simple change of coordinates, the de Sitter solution could be interpreted as an expansion of the system of coordinates in hyperbolic space (Lanczos 1922).

$$
\mathrm{d} s^{2}=-\mathrm{d} t^{2}+\cosh ^{2} t\left[\mathrm{~d} \phi^{2}+\cos ^{2} \phi\left(\mathrm{d} \psi^{2}+\cos ^{2} \psi \mathrm{d} \chi^{2}\right)\right]
$$

Lanczos wrote that:

It is interesting to observe how one and the same geometry can appear with quite different physical interpretations according to the interpretations placed upon the particular coordinates.

At almost exactly the same time, the Soviet meteorologist and theoretical physicist Alexander Alexandrovich Friedman published the first of his two classic papers on relativistic cosmology (Friedman 1922, 1924). His key realization was that isotropic world models had to have isotropic curvature everywhere. In the paper of 1922, Friedman found solutions for expanding world models with closed spatial geometries, including those that expand to a maximum radius and then collapse to a singularity. In the paper of 1924, he showed that 


\section{S. Longair}

there exist expanding solutions that are unbounded with hyperbolic geometry. The differential equations that he derived were:

$$
\begin{aligned}
& \left(\frac{\dot{R}}{R}\right)^{2}+\left(\frac{2 R \ddot{R}}{R^{2}}\right)+\frac{c^{2}}{R^{2}}-\Lambda=0 \\
& \left(\frac{\dot{R}}{R}\right)^{2}+\left(\frac{2 R \ddot{R}}{R^{2}}\right)-\frac{c^{2}}{R^{2}}-\Lambda=0
\end{aligned}
$$

In both cases,

$$
\frac{3 \dot{R}^{2}}{R^{2}}+\frac{3 c^{2}}{R^{2}}-\Lambda=\kappa c^{2} \rho
$$

The solutions of these equations correspond exactly to the standard world models of general relativity and are appropriately known as the Friedman world models. The history of general relativity in the Soviet Union is a remarkable story and Friedman's role in introducing Soviet scientists to the theory and the subsequent difficult development of these studies in the USSR needs to be better known. It has been carefully described by Zelmanov (1967) in a review that has not been translated into English.

It has always been considered somewhat surprising that it was some years before Friedman's important papers were given the recognition they deserve. In 1923, Einstein believed he had found an error in the first of Friedman's papers and published his concern in Zeitschrift für Physik. Friedman showed that Einstein was incorrect and Einstein subsequently published his withdrawal of his objection in the same journal. My guess is that Einstein's concern was remembered, but not his acknowledgment of his error.

In 1927, Georges Lemaitre independently discovered the Friedman solutions and only then became aware of Friedman's pioneering contributions (Lemaitre 1927). Both Lemaitre and Howard P. Robertson (1928) were aware of the fact that the Friedman solutions result locally in a velocity-distance relation. Lemaitre derived what he termed the "apparent Doppler effect," in which "the receding velocities of extragalactic nebulae are a cosmical effect of the expansion of the Universe" with $v \propto r$. Robertson found a similar result stating that "we should expect ... a correlation $v \approx(c l / R)$," where $l$ is distance and $v$ the recession velocity. From nearby galaxies, he found a value for Hubble's constant of $500 \mathrm{~km} \mathrm{~s}^{-1} \mathrm{Mpc}^{-1}$.

The discovery of the velocity-distance relation for galaxies was interpreted as evidence for the expansion of the Universe as a whole. There remained problems of interpretation of the notions of time and distance in cosmology because the field equations could be set up in any frame of reference. By 1935, the problem had been solved independently by Robertson and George Walker (Robertson 1935; Walker 1935). For isotropic, homogeneous world models, they showed that the metric of spacetime had to have the form

$$
\mathrm{d} s^{2}=\mathrm{d} t^{2}-\frac{R^{2}(t)}{c^{2}}\left[\frac{\mathrm{d} r^{2}}{\left(1+\kappa r^{2}\right)}+r^{2}\left(\mathrm{~d} \theta^{2}+\sin ^{2} \theta \mathrm{d} \phi^{2}\right)\right]
$$

where $\kappa$ is the curvature of space at the present epoch, $r$ is a comoving radial distance coordinate and $R(t)$ is the scale factor which describes how the distance between any two world lines change with cosmic time $t$. The Robertson-Walker metric contains all the geometries consistent with the assumptions of isotropy and homogeneity of the Universe; the curvature $\kappa=\mathcal{R}^{-2}$, where $\mathcal{R}$, the radius of curvature of the spatial sections of the isotropic curved 


\section{S. Longair}

space, can be positive, negative or zero. The physics of the expansion is absorbed into the scale factor $R(t)$.

With the discovery of the velocity-distance relation, Einstein regretted the inclusion of the cosmological constant into the field equations. According to George Gamow, Einstein stated that the introduction of the cosmological constant was "the biggest blunder of my life" (Gamow 1970). In 1932, Einstein and de Sitter showed that there is one special solution of the equations with $\Lambda=0$ and $\kappa=0$, corresponding to Euclidean space sections (Einstein \& de Sitter 1932). This Einstein-de Sitter model has density at the present epoch $\rho_{0}=3 H_{0}^{2} / 8 \pi G$. This density is often referred to as the critical density and the Einstein-de Sitter model as the critical model, because it separates the ever-expanding models with open, hyperbolic geometries from those that will eventually collapse to a singularity and that have closed, spherical geometry. When Einstein and de Sitter inserted $H_{0}=500 \mathrm{~km} \mathrm{~s}^{-1} \mathrm{Mpc}^{-1}$ into the expression for $\rho_{0}$, they found $\rho_{0}=4 \times 10^{-25} \mathrm{~kg} \mathrm{~m}^{-3}$. Although this value was somewhat greater than the mean density in galaxies derived by Hubble, they argued that it was of the correct order of magnitude and that there might well be a considerable amounts of "dark matter" present in the Universe.

\subsection{Astrophysical Cosmology up to 1939}

\subsubsection{Dark Matter}

Astrophysical evidence for dark matter was not long in coming. In 1933, Fritz Zwicky made the first dynamical studies of rich clusters of galaxies, in particular, of the Coma cluster (Zwicky 1933, 1937). The method Zwicky used to estimate the total mass of the cluster involved the virial theorem, which had been derived by Arthur Eddington in 1916 to estimate the masses of star clusters (Eddington 1916). The theorem relates the total internal kinetic energy $T=\frac{1}{2} M\left\langle v^{2}\right\rangle$ of the galaxies in a cluster to its gravitational potential energy, $|U|=G M^{2} / 2 R_{\mathrm{cl}}$ in statistical equilibrium under gravity. Eddington showed that $T=\frac{1}{2}|U|$ and so the mass of the cluster can be found, $M \approx 2 R_{\mathrm{cl}}\left\langle v^{2}\right\rangle / G$.

Zwicky measured the velocity dispersion of the galaxies in the Coma cluster and found that there was much more mass in the cluster than could be attributed to the visible parts of galaxies. In solar units, the ratio of mass to optical luminosity of a galaxy such as our own is about 3, whereas for the Coma cluster the ratio was found to be about 500-there must be about 100 times more dark or hidden matter as compared with visible matter in the cluster. Zwicky's pioneering studies have been confirmed by all subsequent studies of rich clusters of galaxies.

\subsubsection{The Age of the Universe and Eddington-Lemaître Models}

Despite Einstein's renunciation of the cosmological constant $\Lambda$, there remained a very grave problem for those models in which $\Lambda$ is set equal to zero. In all world models with $\Lambda=0$, the age of the Universe is less than $H_{0}^{-1}$. Using Hubble's estimate of $H_{0}=500 \mathrm{~km}$ $\mathrm{s}^{-1} \mathrm{Mpc}^{-1}$, the age of the Universe must be less than $2 \times 10^{9}$ years old, a figure in conflict with the age of the Earth derived from studies of the ratios of abundances of long-lived radioactive species, which gave ages significantly greater than this value.

Eddington and Lemaitre recognized that this problem could be eliminated if $\Lambda$ were positive (Eddington 1930; Lemaître 1931a). The effect of a positive cosmological constant is to counteract the attractive force of gravity when the Universe has grown to a large enough 


\section{S. Longair}

size. Among the solutions of Einstein's equations, there are special cases equivalent to the Einstein static Universe, but at some earlier epoch. These models remained in the static Einstein state for an arbitrarily long period and then expanded away from that state under the influence of the cosmological term. In these Eddington-Lemaitre models, the age of the Universe could be arbitrarily long. As Eddington expressed it, the Universe would have a "logarithmic eternity" to fall back on, and so resolve the conflict between estimates of Hubble's constant and the age of the Earth.

\subsubsection{The Origin of the Chemical Elements}

In the 1930s, there were two reasons why the synthesis of the chemical elements during the early stages of the Friedman world models was taken seriously. Firstly, the chemical abundances of the elements in stars seemed to be remarkably uniform. Secondly, it appeared that the interiors of stars were not hot enough for nucleosynthesis of the chemical elements to take place in their interiors. A starting point for a cosmological solution to this problem was to work out the equilibrium abundances of the elements at some very high temperature and assume that, if the density and temperature decreased sufficiently rapidly, these abundances would remain "frozen."

In 1931, Lemaitre proposed that the initial state of the Friedman models consisted of what he termed a "primaeval atom" (Lemaitre 1931b). Following the discovery of the neutron in the following year, this state could be thought of as a sea of neutrons closely packed together. The primaeval neutrons were supposed to decay into protons and the chemical elements, as well as the cosmic rays, form in the subsequent nuclear interactions. These ideas inspired George Gamow's attack upon the problem of the origin of the chemical elements. In 1946, he extrapolated the Freidman models back to epochs when the densities and temperatures were high enough for nucleosynthesis to take place and found that the time scale of the Universe during these early stages was too short to establish an equilibrium distribution of the elements (Gamow 1946).

\subsection{The Cosmological Problem in 1939}

By the end of the 1930s, there was a common view that the solution of the cosmological problem lay in the determination of the parameters which define the Friedman world models. This became one of the great goals of the programs of observation to be carried out by the Palomar 200-inch telescope (Sandage 1961) and the subsequent generation of 4 -meter class telescopes. The challenge was to measure precisely the parameters that characterize the Universe: Hubble's constant, $H_{0}=\dot{R} / R$; the deceleration parameter, $q_{0}=-\ddot{R} / \dot{R}^{2}$; the curvature of space $\kappa=\mathcal{R}^{-2}$; the mean density of matter in the Universe $\rho$ and, in particular, whether or not it attains the critical density $\rho_{0}$; the age of the Universe, $T_{0}$; and the cosmological constant $\Lambda$. These are not independent. According to general relativity,

$$
\kappa=\mathcal{R}^{-2}=\frac{(\Omega-1)+\frac{1}{3}\left(\Lambda / H_{0}^{2}\right)}{\left(c / H_{0}\right)^{2}} \quad q_{0}=\frac{\Omega}{2}-\frac{1}{3} \frac{\Lambda}{H_{0}^{2}},
$$

where $\Omega=\rho / \rho_{0}$ is known as the density parameter, where $\rho_{0}$ is the critical density. The determination of these parameters turned out to be among the most difficult observational challenges in astronomy, and progress by the traditional techniques of optical astronomy proved to be much more difficult than the optimists of the 1930s must have hoped. The Palomar 200-inch telescope was commissioned in 1948, and much effort was devoted to 


\section{S. Longair}

the determination of cosmological parameters, particularly by Allan Sandage, who has published a splendid review of this heroic endeavor (Sandage 1994).

In compensation, completely new vistas were to open up after the Second World War as the whole of the electromagnetic spectrum became available for astronomical observation and completely new approaches to the determination of cosmological parameters and the origin of structure in the Universe became possible.

\subsection{Post-War Cosmology to 1970}

\subsubsection{Gamow and the Big Bang}

The first detailed calculations of the expected abundances of the elements according to Lemaître's concept of the "primaeval atom" were carried out in 1942 by Chandrasekhar \& Henrick (1942). They confirmed the expectation of equilibrium theory that, if the elements are in equilibrium at temperatures of about $10^{10} \mathrm{~K}$ and densities of about $10^{9} \mathrm{~kg} \mathrm{~m}^{-3}$, their abundances should be inversely correlated with their binding energies. There were, however, several gross discrepancies with the observed abundances. The light elements, lithium, beryllium, and boron, were vastly overproduced and iron and the elements with mass numbers greater than about 70 underproduced. They suggested that some non-equilibrium process was required.

In 1946, George Gamow found that the time scale of early expansion of the Universe was indeed too short to establish an equilibrium abundance of the elements (Gamow 1946). Neutron capture cross sections became available in 1946 as a by-product of the nuclear physics programs carried out during the Second World War, and these showed that there is an inverse correlation between these cross sections and the relative abundances of the elements. In the first calculations carried out by Gamow and Ralph Alpher, the computations assumed a sea of free neutrons and that nucleosynthesis only took place after the temperature had fallen below $k T=0.1 \mathrm{MeV}$-the Universe was assumed to be static. This theory was published in 1948 by Alpher, Bethe, \& Gamow (1948) and they found reasonable agreement with the observed abundances of the elements. The paper drew attention to the necessity of a hot, dense phase in the early Universe if the elements were to be synthesized cosmologically.

In the same year, Alpher and Robert Herman (1948) carried out improved calculations, including the cosmic expansion into their calculations. They realised that, at such very high temperatures at early epochs, the Universe was radiation rather than matter-dominated and they solved the problem of the subsequent temperature history of the Universe. They came to the far-reaching conclusion that the cooled remnant of the hot early phases should be present in the Universe today and estimated that the temperature of this thermal background should be about $5 \mathrm{~K}$.

There was, however, a major problem with this picture-there are no stable nuclei with mass numbers 5 and 8 . Fermi and Turkevich calculated the evolution of the nuclear abundances of the light elements including 28 nuclear reactions for elements up to mass number 7 in a radiation-dominated, expanding Universe and their results were published by Alpher \& Herman (1950). These calculations showed that only about one part in $10^{7}$ of the initial mass was converted into elements heavier than helium.

In 1950, Hayashi pointed out that, in the early Universe, at temperatures only ten times greater than that at which the nucleosynthesis took place, the neutrons and protons were 


\section{S. Longair}

brought into thermal equilibrium by the weak interactions:

$$
\mathrm{e}^{+}+\mathrm{n} \leftrightarrow \mathrm{p}+\bar{\nu}_{\mathrm{e}} \quad \nu_{\mathrm{e}}+\mathrm{n} \leftrightarrow \mathrm{p}+\mathrm{e}^{-}
$$

(Hayashi 1950). At about the same temperature, electron-positron pair production ensures a plentiful supply of positrons and electrons. Thus, rather than assume arbitrarily that the initial conditions consisted of a sea of neutrons, the equilibrium abundances of protons, neutrons, electrons and all the other constituents of the early Universe could be calculated exactly. In 1953, Alpher, Follin, \& Herman (1953) determined the evolution of the protonneutron ratio as the Universe expanded and obtained an answer remarkably similar to modern calculations. These ideas were, however, overtaken by the discovery of the physics of nucleosynthesis of the chemical elements in stars.

\subsubsection{Steady-state Cosmology}

Immediately after the War, many new ideas were in the air. Milne had developed his theory of kinematic relativity, in which there are two times, one associated with dynamical phenomena and another with electromagnetic phenomena (Milne 1948). Dirac had been impressed by coincidences between the very large numbers in physics and the properties of the Universe-for example, the square of the ratio of the strengths of electromagnetic and gravitational forces is roughly equal to the numbers of protons in the Universe (Dirac 1937). A consequence of his identification of these large numbers was the idea that the gravitational constant should change with time. Eddington had developed his Fundamental Theory, in which the cosmological constant appeared as a fundamental constant of nature (Eddington 1946).

Steady-state cosmology was invented by Hermann Bondi, Thomas Gold and Fred Hoyle in 1948 (Bondi \& Gold 1948; Hoyle 1948). They extended the cosmological principle to what they termed the perfect cosmological principle according to which the Universe presents the same large-scale picture to all fundamental observers at all times. Hence, Hubble's constant becomes a fundamental constant of nature. The perfect cosmological principle led to a unique metric for the dynamics of the Universe with zero spatial curvature. Because of the expansion of the Universe, matter has to be continuously created in order to replace the dispersing matter, the rate of creation amounting to only one particle $\mathrm{m}^{-3}$ every 300,000 years. A consequence of the theory was that the Universe was infinite in age, but the age of typical objects observed in the local Universe is only $\frac{1}{3} H_{0}^{-1}$. It was during a radio program on cosmology in the late 1940s that Hoyle introduced the somewhat pejorative term "Big Bang" to describe the Friedman models with singular origin, which is eliminated in the steady-state picture.

Hoyle set about finding an alternative means of understanding the formation of the chemical elements by nucleosynthesis in stars and these considerations led to his remarkable prediction of the carbon resonance (Hoyle 1953) for the formation of carbon in stars and the important paper on the processes of stellar nucleosynthesis by Burbidge, Burbidge, Fowler, $\&$ Hoyle (1957). With these new insights, the abundances of the chemical elements disappeared as evidence for a hot initial phase of the Universe.

In the 1950s, two important results were reported of central importance for cosmology. The first concerned the value of Hubble's constant. At the meeting of the International Astronomical Union in Rome in 1952, Walter Baade announced that the distance to the Andromeda Nebula (M31) had been underestimated by a factor of 2 (Baade 1952). Hubble's 


\section{S. Longair}

constant was therefore reduced to $250 \mathrm{~km} \mathrm{~s}^{-1} \mathrm{Mpc}^{-1}$ and $H_{0}^{-1}$ increased to $4 \times 10^{9}$ years. In 1956, Humason, Mayall, \& Sandage (1956) revised Hubble's constant downward again to $180 \mathrm{~km} \mathrm{~s}^{-1} \mathrm{Mpc}^{-1}$. These revisions eliminated the discrepancy between the age of the Earth and the age of the Universe according to the Friedman models with $\Lambda=0$. By the 1970s, the value was reduced further to between 50 and $100 \mathrm{~km} \mathrm{~s}^{-1} \mathrm{Mpc}^{-1}$. The precise value became a subject of considerable controversy, but this has now been resolved, largely thanks to the leadership of the Hubble Key Program by Dr. Wendy Freedman, the chair of this Symposium (Freedman et al 2001).

The second concerned the number counts of radio sources that showed that there was an excess of faint radio sources. Martin Ryle concluded that the only reasonable interpretation was that there was a much greater comoving number density of extragalactic radio sources at large distances, and hence at earlier cosmic epochs, than nearby. As Ryle expressed it in his Halley Lecture in 1955, "there seems no way in which the observations can be explained in terms of a Steady-State theory" (Ryle 1955). This led to a somewhat bitter controversy, both within the radio astronomical community and with the proponents of the steady-state theory. By the 1960s, it was established that Ryle's conclusion was correct, but the effect was not nearly as large as had been believed in the 1950s, because the importance of source confusion had not been appreciated.

Although steady-state theory is nowadays considered to be largely of historical and scientific sociological interest, there are some features of the theory that have a resonance with contemporary cosmological theories. In the steady-state picture,

- The density of the Universe is a constant

- The spatial geometry is flat

- The scale factor varies as $\exp \left\{H_{0}\left(t_{0}-t\right)\right\}$

These features have a rather familiar ring about them nowadays and correspond rather precisely to the present best-buy picture of the Universe, in which we are entering a phase when its dynamics are to be dominated by the dark energy, equivalent to the presence of a significant cosmological constant $\Lambda$. In Hoyle's version of steady-state theory, these properties are attributed to the action of the creation field $C$. It is amusing to note that on the occasion of Fred's 80th birthday celebrations in 1995, he gave a splendid lecture to the Cavendish Physical Society in which he stated that, if only he had called the creation field $\psi$, rather than $C$, he would now would be remembered as the originator of the inflationary Universe.

William McCrea had, however, already had this deep insight in 1951 (McCrea 1951). McCrea realised that there was a quite different interpretation of what Hoyle had done, which bears a much closer resonance with contemporary cosmology. To quote McCrea,

The single admission that the zero of absolute stress may be set elsewhere than is currently assumed on somewhat arbitrary grounds permits all of Hoyle's results to be derived within the system of general relativity theory. Also, this derivation gives the results an intellectual physical coherence.

McCrea wrote the physics of the steady-state picture in terms of a negative energy equation of state $p=-\rho c^{2}$ and recovered the three features of the theory listed above. It is intriguing that McCrea had realised that there is nothing intrinsically implausible about a negative energy equation of state. Indeed, this is what we believe dominates the dynamics of the Universe from now on. 


\section{S. Longair}

\subsection{The Helium Problem and the Microwave Background Radiation}

In 1961 Osterbrock \& Rogerson (1961) showed that the fractional abundance of helium seemed to be remarkably uniform wherever it could be observed and corresponded to about 25\% by mass. In 1964 O'Dell, Peimbert, \& Kinman (1964) found that the helium abundance in a planetary nebula in the old globular cluster M15 also had helium abundance about $25 \%$, despite the fact that the heavy elements were very significantly depleted relative to their cosmic abundances.

It is not often that a fundamental aspect of contemporary cosmology is developed in the course of a post-graduate lecture course, but this in fact occurred in the solution of the helium problem. In the Lent term of 1964, my first year as a research student in Cambridge, Fred Hoyle gave a post-graduate lecture course entitled Extragalactic Astrophysics and Cosmology. It was given twice a week to a remarkable group of research students, many of whom went on to become leaders of astronomy. Fred would turn up with a few notes scribbled on what looked like the traditional envelope and run through what indeed turned out to be many of the key problems of astrophysics during the subsequent decades.

Toward the end of the course, he tackled the problem of the origin of helium in the cosmos, reviewing the early work of Alpher, Gamow and Hermann. Roger Tayler and John Falconer were in the audience, and they realised that they could use the EDSAC-2 computer to carry out predictions of the cosmic helium abundance for a wide range of different cosmological models. In the course of the following two lectures, they unraveled in some detail the implications of these calculations, and the result was the famous Nature paper by Hoyle \& Tayler (1964), which revived interest in the primordial synthesis of the light elements. They found that about $25 \%$ helium by mass is synthesised in the Big Bang, in remarkable agreement with observation, and that this result is essentially independent of the present baryonic density. Hoyle and Tayler did not mention that the cooled remnant of the hot early Universe should be detectable at centimeter wavelengths. Alpher and Herman's prediction had been more or less forgotten when Gamow's theory of primordial nucleosynthesis had failed to account for the creation of the elements.

In the very next year 1965, the microwave background radiation was discovered, more or less by accident, by Arno Penzias and Robert Wilson (1965). During the commissioning of a 20 -foot horn antenna designed for telecommunications, they found an excess noise temperature of about $3.5 \pm 1 \mathrm{~K}$ wherever they pointed their telescope at the sky. Robert Dicke's group in Princeton was attempting exactly this experiment to detect the cooled remnant of the Big Bang - it was very quickly realised that Penzias and Wilson had discovered the signal sought by the Princeton physicists. Within a few months, the Princeton group had measured a background temperature of $3.0 \pm 0.5 \mathrm{~K}$ at a wavelength of $3.2 \mathrm{~cm}$, confirming the black body nature of the background spectrum (Roll \& Wilkinson 1966).

\subsection{Conclusions}

This seems an appropriate point at which to conclude this brief review. By the late 1960s and early 1970s, the observational evidence strongly favored what has become the standard Big Bang framework for contemporary geometrical and astrophysical cosmology. The 1960s and 1970s were also decades during which the whole face of astronomy, astrophysics and cosmology were revitalized through the opening up of the complete electromagnetic spectrum for astronomical observation. Some highlights of these observational advances would include: 


\section{S. Longair}

- the discovery of quasars

- the strong cosmological evolution of all classes of active galaxies

- the discovery of deuterium in the interstellar medium

- the discovery of gravitational lensing

- the discovery of the diffuse $\mathrm{X}$-ray emission from clusters of galaxies

- the observation of the Sunyaev-Zel'dovich effect

- $C O B E$ observations of the spectrum and fluctuations in the cosmic microwave background radiation

- the discovery of the Type Ia supernova technique for estimating cosmological parameters

- precise measurements of the distances of nearby galaxies

- the determination of the mean mass density of the Universe on large scales

A corresponding list could be drawn up for theoretical advances:

- the determination of the detailed thermal history of the Universe

- the theory of the development of fluctuations in standard Big Bang picture and the resulting prediction of the spectrum of fluctuations in cosmic microwave background radiation

- the mathematical analysis of the large-scale structure of the Universe

- constraints on the number of neutrino species and the early dynamics of the Universe from primordial nucleosynthesis

- the cold dark matter scenario for the origin of structure

- the inflationary scenario for the very early Universe

- the use of massive parallel computing to simulate the origin of large-scale structures in the Universe

All these topics are now the bread and butter of modern cosmology and will be discussed in extenso during this centennial meeting. It is startling to realise just how far we have come in the matter of only 30 years. Few of us who began our research careers in the early 1960s could have predicted the enormous advances in observational and theoretical cosmology, far less the extraordinary fact that there seems to be a concordance between the many different approaches to geometrical and astrophysical cosmology. None of this could have come about without the pioneering efforts of many great astronomers whose endeavors have been the theme of this survey. In turn, these astronomers could not have made their discoveries without the tools provided by generous benefactors of astronomy. Among these, Andrew Carnegie's name will always be remembered as the founder of the Observatories of the Carnegie Institution of Washington, which has been, and continues to be, at the forefront of the best of contemporary astronomy and cosmology. 


\section{S. Longair}

\section{References}

\section{General References}

Many important topics are mentioned only briefly in this short survey. I have found the following books particularly helpful in preparing this paper.

Bernstein, J., \& Feinberg, G. 1986, Cosmological Constants: Papers in Modern Cosmology (New York: Columbia Univ. Press)

Bertotti, B., Balbinot, R., Bergia, S., \& Messina, A., ed. 1990, Modern Cosmology in Retrospect (Cambridge: Cambridge Univ. Press)

Bondi, H. 1960, Cosmology, 2nd edition (Cambridge: Cambridge Univ. Press)

Gillespie, C. C., ed. 1981, Dictionary of Scientific Biography (New York: Charles Scribner's Sons)

Gingerich, O., ed. 1984, The General History of Astronomy, Vol. 4., Astrophysics and Twentieth-Century Astronomy to 1950: Part A (Cambridge: Cambridge Univ. Press)

Harrison, E. 2001, Cosmology: The Science of the Universe (Cambridge: Cambridge Univ. Press)

Hearnshaw, J. B. 1986, The Analysis of Starlight: One Hundred and Fifty Years of Astronomical Spectroscopy (Cambridge: Cambridge Univ. Press)

- 1996, The Measurement of Starlight: Two Centuries of Astronomical Photometry (Cambridge: Cambridge Univ. Press)

Lang, K. R., \& Gingerich, O., ed. 1979, A Source Book in Astronomy and Astrophysics, 1900-1975 (Cambridge, Mass.: Harvard Univ. Press)

Learner, R. 1981, Astronomy Through the Telescope (London: Evans Brothers Limited)

Leverington, D. 1996, A History of Astronomy from 1890 to the Present (Berlin: Springer-Verlag)

Longair, M. S. 1995, Astrophysics and Cosmology, in 20th Century Physics, 3, ed. L. M. Brown, A. Pais, \& A. B. Pippard (Bristol: Institute of Physics Publications: New York: American Institute of Physics), 1691-1821. A much expanded version of this text will be published by Cambridge University Press in 2003.

Martínez, V. J., Trimble, V. \& Pons-Bordeía, M. J., ed. 2001, Historical Development of Modern Cosmology (San Francisco: ASP), 252

North, J. D. 1965, The Measure of the Universe (Oxford: Clarendon Press)

Literature References

Alpher, R. A., Bethe, H., \& Gamow, G. 1948, Phys. Rev., 73, 803

Alpher, R. A., Follin, J. W., \& Herman, R. C. 1953, Phys. Rev., 92, 1347

Alpher, R. A., \& Herman, R. C. 1948, Nature, 162, 774

. 1950, Rev. Mod. Phys., 22, 153

Baade, W. 1952, Trans. IAU, 8, 397

Bondi, H., \& Gold, T. 1948, MNRAS, 108, 252

Burbidge, E. M., Burbidge, G. R., Fowler, W. A., \& Hoyle, F. 1957, Rev. Mod. Phys., 29, 547

Chandrasekhar, S., \& Henrick, L. R. 1942. ApJ, 95, 288

de Sitter, W. 1917, MNRAS, 78, 3

Dirac, P. A. M. 1937, Nature, 139, 323

Eddington, A. S. 1916, MNRAS, 76, 525

-. 1930, MNRAS, 90, 669

—. 1946, Fundamental Theory, ed. E. Whittaker (Cambridge: Cambridge Univ. Press)

Einstein, A. 1915, Sitzungsberichte Preuss. Akad.Wissenschaften, 844

—. 1917, Sitzungsberichte Berl. Akad., 1, 142

Einstein, A., \& de Sitter, W. 1932, Proc. Natl. Acad. Sciences, 18, 213

Freedman, W. L., et al. 2001, ApJ, 533, 47

Friedman, A. A. 1922, Zeitschrift für Physik, 10, 377

—. 1924, Zeitschrift für Physik, 21, 326

Gamow, G. 1946, Phys. Rev., 70, 572

1970, My World Line (New York: Viking Press), 44

Hayashi, C. 1950, Prog. Theor. Phys. (Japan), 5, 224

Hoyle, F. 1948, MNRAS, 108, 372

1953, ApJS, 1, 121

Hoyle, F., \& Tayler, R. J. 1964, Nature, 203, 1108 


\section{S. Longair}

Hubble, E. P. 1925, Publ. Amer. Astron. Soc., 5, 261

- 1926, ApJ, 64, 321

- 1929, Proc. Natl. Acad. Sciences, 15, 168

Hubble, E. P., \& Humason, M. 1934, ApJ, 74, 43

Humason, M. L., Mayall, N. U., \& Sandage, A. R. 1956, ApJ, 61, 97

Lanczos, C. 1922, Phys. Zeitschrift, 23, 539

Leavitt, H. S. 1912, Harvard College Observatory Circular, No. 173, 1

Lemâitre, G. 1927, Annales Société Scientifique de Bruxelles, A47, 49

-. 1931a, MNRAS, 91, 483

-. 1931b, Nature, 127, 706

Lobachevsky, N. I. 1829-30, On the Principles of Geometry (Kazan Bulletin)

Longair, M. S. 2001, in Historical Development of Modern Cosmology, ed. V. J. Martínez, V. Trimble, \&

M. J.Pons-Bordeía, (San Francisco: ASP), 55

McCrea, W. H. 1951, Proc. Roy. Soc., 206, 562

Milne, E. 1948, Kinematic Relativity (Oxford: Clarendon Press)

O’Dell, C. R., Peimbert, M., \& Kinman, T. D. 1964, ApJ, 140, 119

Osterbrock, D. E. 1984, James E. Keeler: Pioneer American Astrophysicist (Cambridge: Cambridge Univ. Press)

Osterbrock, D. E., \& Rogerson, J. B. 1961, PASP, 73, 129

Penzias, A. A., \& Wilson, R. W. 1965, ApJ, 142, 419

Robertson, H. P. 1928. Phil. Mag., 5, 835

1935, ApJ, 82, 284

Roll, P. G., \& Wilkinson, D. T. 1966, Phys. Rev. Lett., 16, 405

Ryle, M. 1955, Observatory, 75, 137

Sandage, A. R. 1961, ApJ, 133, 355

. 1994, in The Deep Universe, ed. B. Binggeli \& R. Buser (Berlin: Springer-Verlag), 1

Slipher, V. M. 1917, Proc. Amer. Phil. Soc., 56, 403

Walker, A. G. 1936, Proc. Lond. Math. Soc., Ser. 2., 42, 90

Wirtz, C. W. 1921, Astronomische Nachrichten, 215, 349

Wright, H., Warnow, J. N., \& Weiner, C., ed. 1972, The Legacy of George Ellery Hale (Cambridge, Mass: MIT Press)

Zel'dovich, Ya. B. 1968, Uspekhi Fizicheskikh Nauk, 95, 209

Zelmanov, A. 1967, in Development of Astronomy in the USSR, ed V. A. Ambartsumian et al. (Moscow: Nauka), 320

Zwicky, F. 1933, Helv. Physica Acta, 6, 110

—. 1937, ApJ, 86, 217 